\title{
475528 - DEFINING RULES FOR THE IDENTIFICATION OF VENTILATORY EVENTS
}

\author{
Maryam Dosani, B.Sc., Erica Amari, BA, Peter Choi, MD, Stephan Schwarz, M.D., \\ Ph.D., FRCPC, J Mark Ansermino, FRCPC \\ Anesthesiology, Pharmacology \& Therapeutics, University of British Columbia, \\ Vancouver, BC, Canada
}

Introduction: The automated recognition of significant clinical events by physiological monitors is a challenging task, exacerbated by a lack of standardized and clinically relevant threshold criteria. Our objective was to develop consensus regarding the thresholds used to identify three ventilatory events: disconnection or significant leak in the anesthesia circuit, decreased pulmonary compliance or increased resistance, and inhaled anesthetic overdose.

Methods: After ethics committee approval, we conducted individual structured interviews with five expert anesthesiologists to gain insight into the cognitive processes used by clinicians to diagnose ventilatory events and to determine the common heuristics (rules of thumb) used in clinical practice. Using ATLAS/ti (T Muhr, Germany, 1994), we analyzed the interview data for common themes which we used to generate items for three web-based questionnaires, each containing 9 to 32 items with 3 to 7 response options per item. Each questionnaire was administered to 18 to 24 experienced anesthesiologists from 11 Canadian institutions, based on a modified two-stage Delphi technique, ${ }^{1}$ to gain consensus on the thresholds for the specific rules identified in the interviews. Responses were collapsed to a specific threshold to achieve a $75 \%$ consensus in median responses. When the median lay between two response options, the clinically conservative response was chosen.

Results: Iterative questionnaire administration indicating participants’ previous responses as well as the group median response facilitated rapid consensus development. Table 1 shows the results from 6 of the 55 items. Agreement was easily achieved for commonly used thresholds (e.g. peak airway pressure) but consensus on a single response option was more difficult to achieve for items that combined information from multiple variables or that identified clinically relevant percentage changes in a single variable. Discussion: This investigation has provided a set of thresholds for developing rules to identify three significant ventilatory events and insight into the decision heuristics used by clinicians in everyday practice.

References: 1 Int J Qual Health Care 1998;10;247-60.

Table 1 Results for 6 items (disconnection or significant leak in the anesthesia circuit questionnaire). 


\begin{tabular}{|c|c|c|c|c|c|}
\hline Questionnaire Item & $\begin{array}{l}\text { Round } 1 \\
\text { Median } \\
\text { Response }\end{array}$ & $\begin{array}{l}\text { \% Who } \\
\text { Chose this } \\
\text { Response }\end{array}$ & $\begin{array}{c}\text { Round } 2 \\
\text { Median } \\
\text { Response }\end{array}$ & $\begin{array}{l}\text { \% Who } \\
\text { Chose this } \\
\text { Response }\end{array}$ & $\begin{array}{l}\text { Value With at } \\
\text { Least } 75 \% \\
\text { Agreement }\end{array}$ \\
\hline \multicolumn{6}{|c|}{ In a healthy ventilated adult using a $3 \mathrm{~L}$ fresh gas flow rate: } \\
\hline $\begin{array}{l}\text { What end tidal } \mathrm{CO}_{2} \text { level } \\
\text { would indicate a DEFINITE } \\
(95 \% \text { chance) leak or } \\
\text { disconnection? }\end{array}$ & $0 \mathrm{mmHg}$ & 61.1 & $0 \mathrm{mmHg}$ & 81.2 & $0 \mathrm{mmHg}$ \\
\hline $\begin{array}{l}\text { What end tidal } \mathrm{CO}_{2} \text { level } \\
\text { would indicate a POSSIBLE } \\
\text { (50\% chance) leak or } \\
\text { disconnection? }\end{array}$ & $20 \mathrm{mmHg}$ & 64.7 & $20 \mathrm{mmHg}$ & 87.5 & $20 \mathrm{mmHg}$ \\
\hline $\begin{array}{l}\text { What DROP in } \mathrm{CO}_{2} \text { would } \\
\text { indicate a DEFINITE ( } 95 \% \\
\text { chance) leak or } \\
\text { disconnection? Initial } \mathrm{CO}_{2} \\
\text { level: } 40\end{array}$ & $40 \%$ & 82.4 & $40 \%$ & 87.5 & $40 \%$ \\
\hline $\begin{array}{l}\text { What DROP in } \mathrm{CO}_{2} \text { would } \\
\text { indicate a POSSIBLE ( } 50 \% \\
\text { chance) leak or } \\
\text { disconnection? Initial } \mathrm{CO}_{2} \\
\text { level: } 40\end{array}$ & $30 \%$ & 70.6 & $30 \%$ & 75.0 & $30 \%$ \\
\hline $\begin{array}{l}\text { What DURATION of end } \\
\text { tidal } \mathrm{CO}_{2} \text { (less than } \\
10 \mathrm{mmHg} \text { ) would indicate a } \\
\text { DEFINITE ( } 95 \% \text { chance) } \\
\text { leak or disconnection? }\end{array}$ & $20 \mathrm{~s}$ & 55.6 & $20 \mathrm{~s}$ & 68.7 & $20 \mathrm{~s}$ \\
\hline $\begin{array}{l}\text { What DURATION of a } \\
\text { change in } \mathrm{CO}_{2} \text { would } \\
\text { indicate a POSSIBLE ( } 50 \% \\
\text { chance) leak or } \\
\text { disconnection? }\end{array}$ & $10 \mathrm{~s}$ & 82.4 & $10 \mathrm{~s}$ & 81.2 & $10 \mathrm{~s}$ \\
\hline
\end{tabular}

